\title{
Acute placentitis and spontaneous abortion caused by Chlamydia psittaci of sheep origin: a histological and ultrastructural study
}

\author{
SY WONG, ${ }^{*}$ ES GRAY,${ }^{*}$ D BUXTON,† J FINLAYSON,† FWA JOHNSON $\ddagger$ \\ From the *Department of Pathology, University Medical Buildings, Foresterhill, Aberdeen, the $\dagger$ Animal \\ Diseases Research Association, Moredun Institute, Edinburgh, and the $\ddagger$ Department of Veterinary Preventive \\ Medicine, Field Station, Leahurst, Neston, Wirral
}

SUMMARY A sheep farmer's wife who had been assisting with lambing developed an influenza like illness in the 28th week of pregnancy. After five days of malaise she spontaneously delivered a stillborn infant; she became acutely ill during the immediate postpartum period with septicaemic shock, acute renal failure, and disseminated intravascular coagulation. The diagnosis was made by isolation and identification of Chlamydia psittaci from the placenta, fetal heart blood, and fetal lung, together with maternal serological evidence. The prominent histological and ultrastructural appearances of the chlamydial placentitis are described.

In the United Kingdom, ovine Chlamydia psittaci (group B chlamydiae) is a major cause of abortion in sheep.' Anecdotal reports ${ }^{2-4}$ have suggested that the same group B chlamydiae may also be transmitted to pregnant women and cause abortion. Although chlamydial organisms were isolated from the products of human abortion by Schachter, ${ }^{5}$ no direct causal relation between the agent and the abortion was convincingly shown. The case reported here appears to be the first in which ovine $C$ psittaci has been definitely shown to have caused human abortion. The clinical, microbiological, and serological findings have already been reported ${ }^{6}$ and the purpose of this communication is to record in detail the histological and ultrastructural appearances of human chlamydial placentitis.

\section{Case report}

A previously healthy 28 year old farmer's wife with a history of one previous normal pregnancy was admitted to the Aberdeen Maternity Hospital in the 28th week of pregnancy after spontaneous delivery of a stillborn baby boy at home, which was followed by postpartum haemorrhage. Some weeks before admission she had assisted with the lambing on her

Accepted for publication 7 February 1985 husband's farm, on which some ewes had aborted. For the five days before her own abortion she had had an influenza like illness with fever. Shortly after admission she became acutely shocked and rapidly developed acute renal failure and disseminated intravascular coagulation. She was transferred to the intensive care unit of Aberdeen Royal Infirmary. A chest radiograph showed pulmonary oedema and mild transient pleural effusions with no evidence of pneumonic consolidation. Ultrasonography revealed a bulky uterus, but no evidence of retained products, together with enlargement of the liver and spleen. The patient was treated empirically with intravenous broad spectrum antibiotics, comprising penicillin, cloxacillin, and cefoxitin; she gradually recovered during the next 10 days.

\section{MICROBIOLOGY}

Micro-organisms were not isolated from cultures of blood, urine, throat swabs, and vaginal swabs. Leptospirosis, listeriosis, brucellosis, toxoplasmosis, Q fever, and mycoplasmal infection were all specifically excluded. $C$ psittaci was cultured from the fetal liver, lung, and heart blood and from the placenta. The isolates had the cultural and nutritional characteristics of an ovine rather than an avian strain of $C$ psittaci. $C$ trachomatis was not isolated from maternal cervical or high vaginal swabs or from fetal tissues and placenta.

Between the fifth and eighth days of the illness 


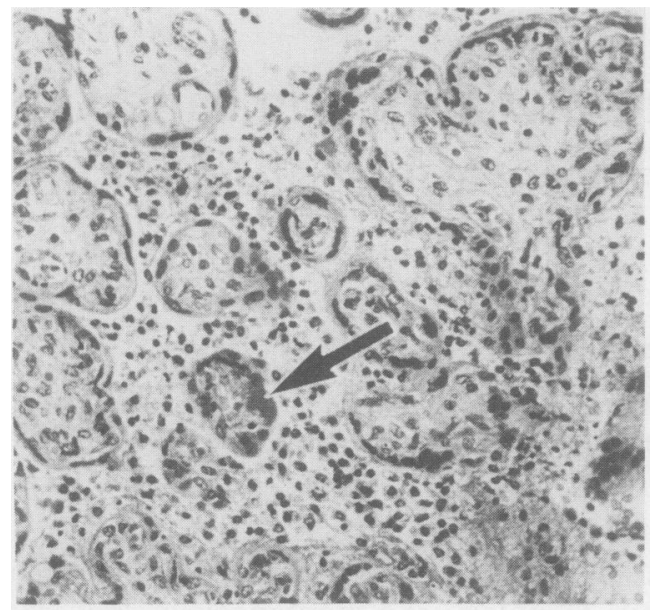

Fig. 1 Human placenta. There is an acute placentitis with a prominent intervillous acute inflammatory exudate. The trophoblast of one villus contains an inclusion (arrowed). Haematoxylin and eosin. $\times 240$.

serological studies on maternal serum samples showed a rise in the titre of complement fixing antibody against chlamydial group antigen prepared from ovine strains of $C$ psittaci from 1/64 to 1/512. In addition, rising titres of neutralising antibody were shown against the chlamydiae which had been isolated from the placenta and fetal tissues. These rising titres were detected against an ovine, but not an avian, strain of $C$ psittaci. Furthermore,

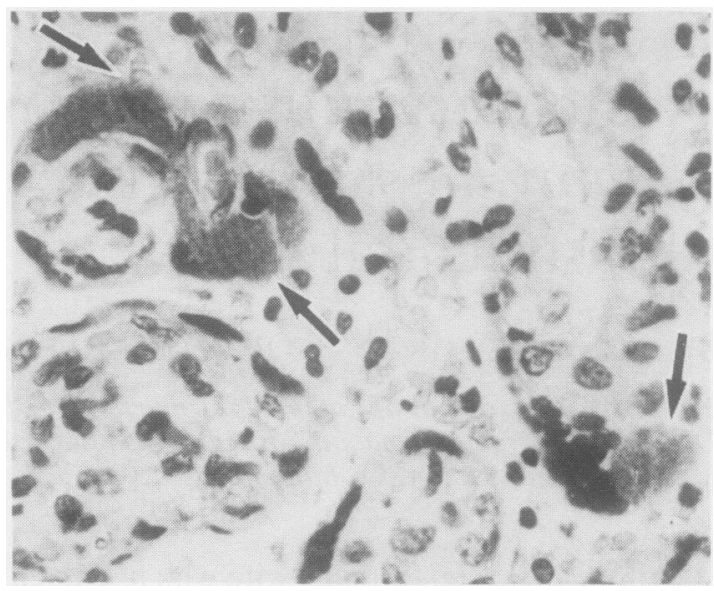

Fig. 2 Three granular, basophilic inclusions (arrowed) in the cytoplasm of the trophoblast. Haematoxylin and eosin. $\times 640$.

immunofluorescence tests showed rising titres of both IgG and IgM antibody to a strain of $C$ psittaci derived from a case of ovine abortion, but only a weak response to a genital (D-K) strain of C trachomatis.

\section{PATHOLOGY}

Necropsy of the stillborn baby boy was performed about $48 \mathrm{~h}$ after death. Externally, the body (weighing $1340 \mathrm{~g}$ ) was that of a premature, well nourished, non-macerated baby of 28 weeks' gestation, with no

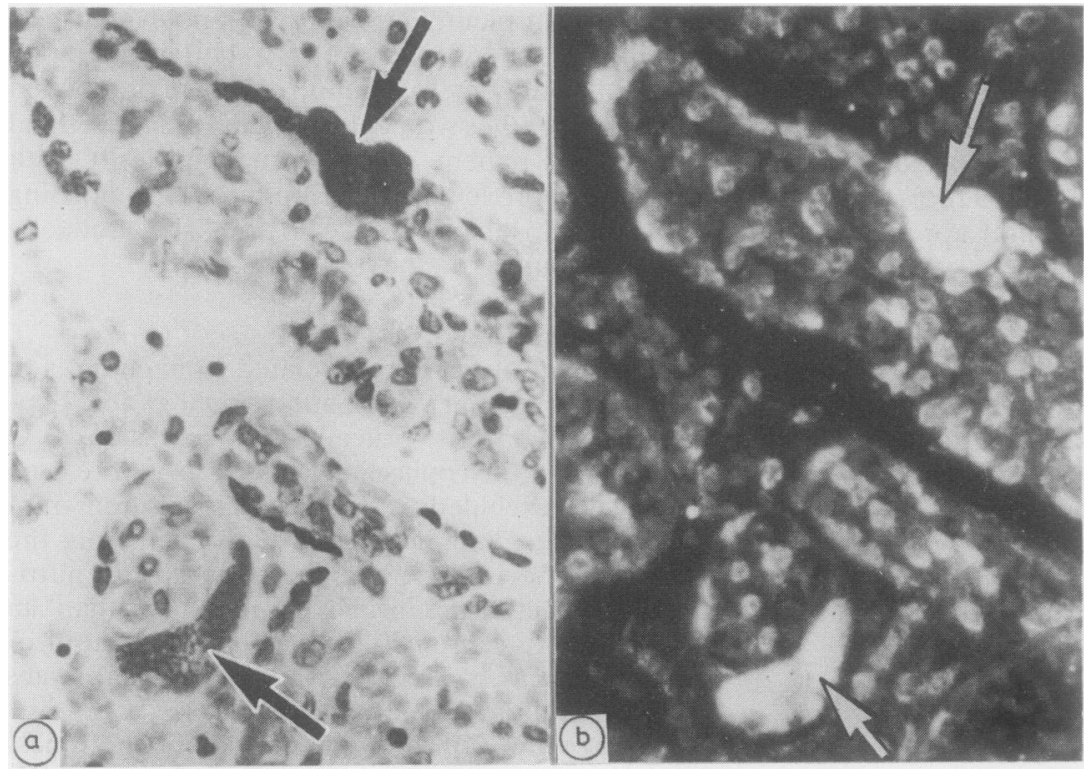

Fig. 3 Inclusions (arrowed) stained by Giemsa and seen by direct light (a) and by dark ground microscopy (b). Giemsa. $\times 640$. 


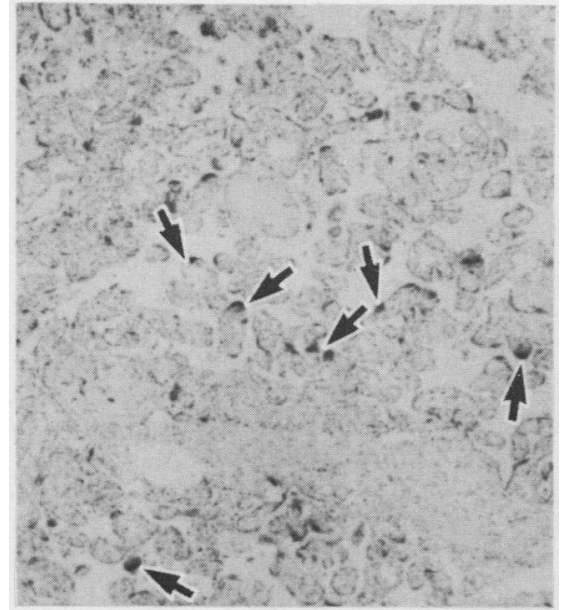

Fig. 4 Direct immunoperoxidase technique using antibody prepared from serum of sheep experimentally infected with ( psittaci, ${ }^{8}$ which binds to the cytoplasmic inclusions (several arrowed) in this placenta tissue section. Direct immunoperoxidase. $\times 40$.

external evidence of disease or malformation. Internally, occasional pinpoint petechial haemorrhages were noted in the thymus $(6 \mathrm{~g})$, on the subpleural surfaces of the lungs, and on the epicardium along the coronary sulcus; such haemorrhages are usually interpreted as being non-specific indicators of terminal anoxia. The lungs (right $16 \mathrm{~g}$, left $14 \mathrm{~g}$ ) were unexpanded. No macroscopic abnormalities were found in the remaining internal organs. The liver $(85 \mathrm{~g})$ and spleen $(3.5 \mathrm{~g})$ were not enlarged. The circular placenta $(215 \mathrm{~g}, 14 \mathrm{~cm} \times 15 \mathrm{~cm})$ showed no gross abnormality of the fetal, maternal, or cut surfaces. The umbilical cord contained three vessels. It was inserted centrally and also appeared normal.

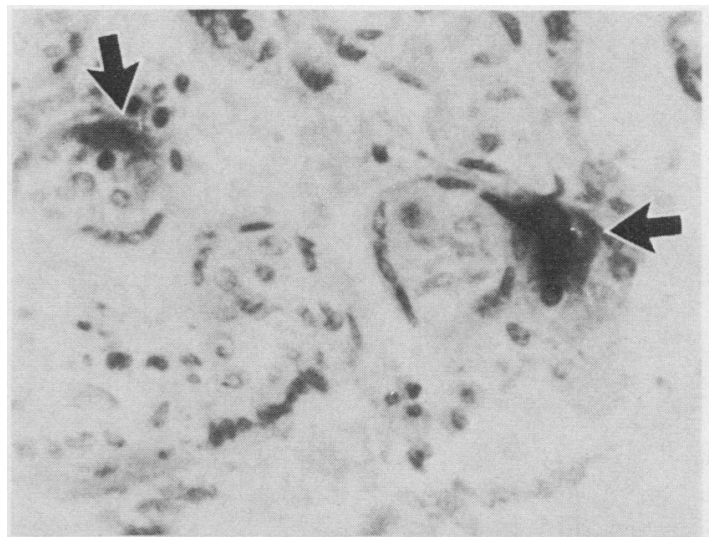

Fig. 5 High power field of the immunoperoxidase stained section clearly defines these chlamydial inclusions (arrowed). Direct immunoperoxidase. Original magnification $\times 640$.

Ovine $C$ psittaci was cultured from both fetal and placental tissue.

Histopathological examination showed that the fetal viscera were unremarkable apart from some cortical atrophy of the thymus gland, which suggested stress in utero for some time before death. The placenta, however, showed a severe placentitis (Fig. 1) with large numbers of acute inflammatory cells in the intervillous spaces together with inflammation of the decidual bed. Occasional fetal stem vessels showed early perivasculitis, but there was no amnionitis. The vessels of the umbilical cord were not affected. In view of the mother's rising antibody titre to chlamydial antigen and the isolation of $C$ psittaci from fetal heart blood and tissues, a careful search was made for histological evidence of chlamydial involvement in the placentitis.

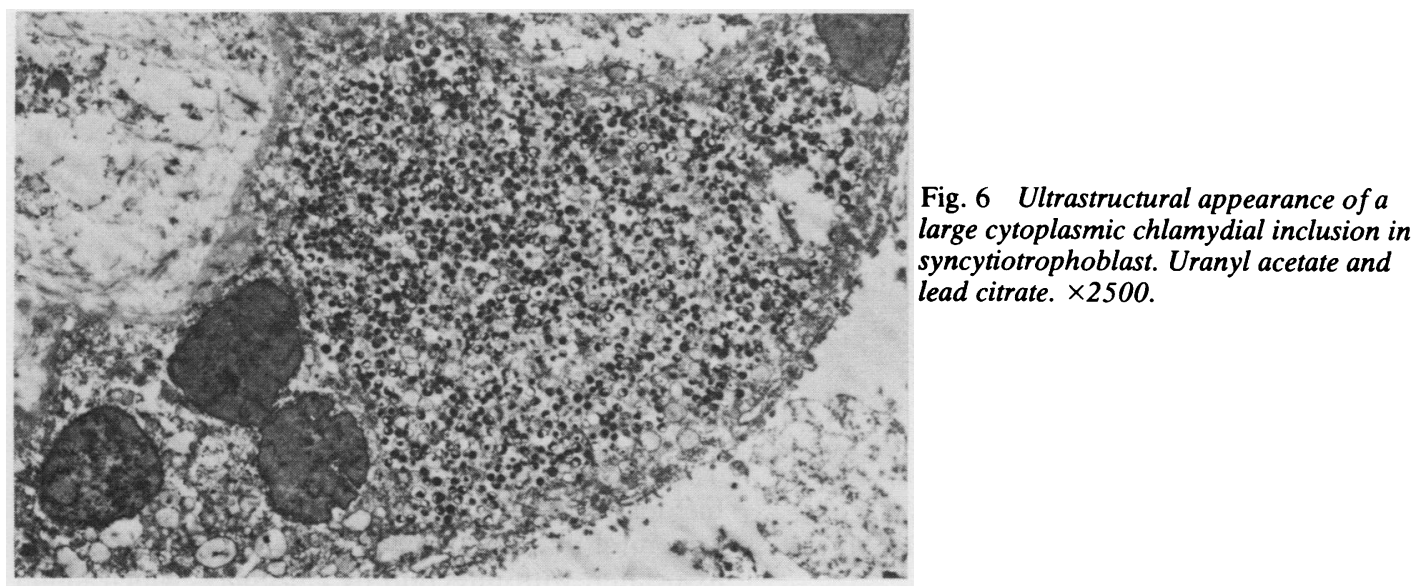




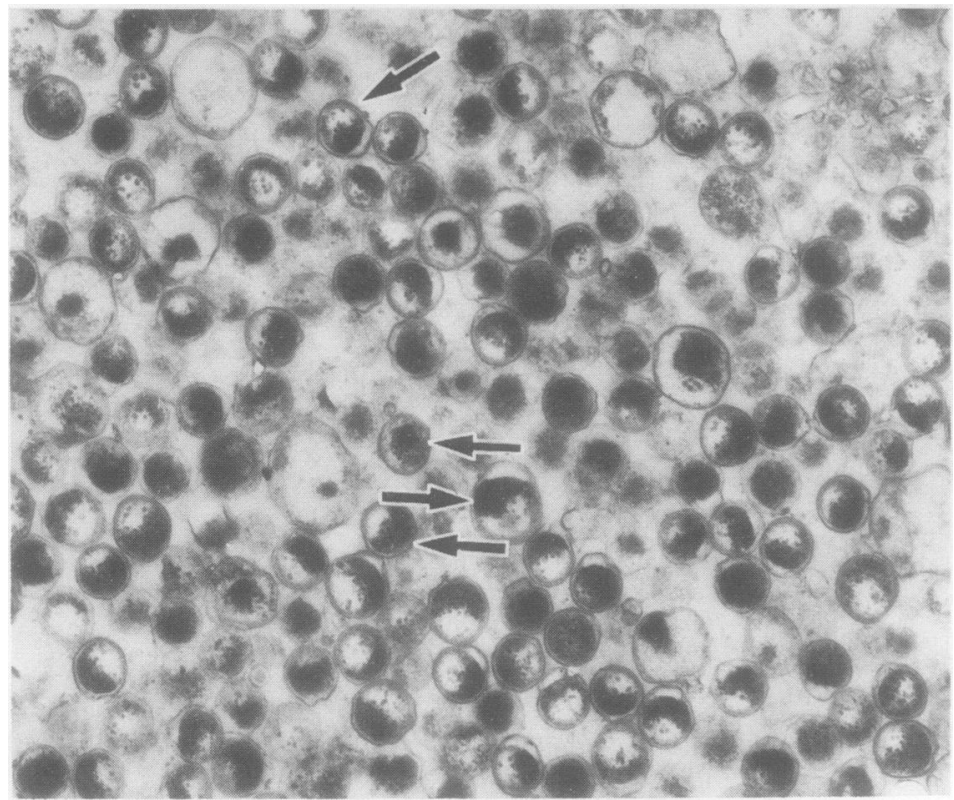

Fig. 7 This chlamydial inclusion is densely packed with a large number of infectious elementary bodies (several arrowed), with occasional intermediate and reticulate forms. No glycogen matrix is present. Uranyl acetate and lead citrate. $\times 15000$.

Haematoxylin and eosin stained sections showed, lightly basophilic, granular inclusions with the morphology of Halberstaeder-Prowazek chlamydial inclusions (Fig. 2). In Giemsa stained sections these inclusions appeared dark blue with transmitted light and, when viewed by dark ground microscopy, brilliant bluish white (Fig. 3). Likewise, with methylene blue staining, ${ }^{7}$ the placental tissue sections showed

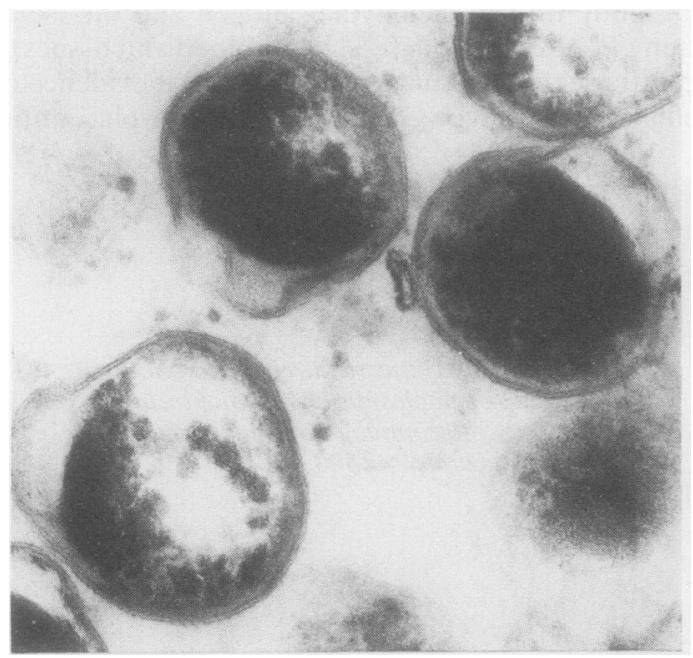

Fig. 8 Each of these chlamydial elementary bodies is enclosed by a rigid cell wall. Uranyl acetate and lead citrate. $\times 75000$. these inclusions as sky blue on direct light microscopy and brilliant greenish blue using dark ground microscopy. In addition, the inclusions stained positively with a direct immunoperoxidase technique recently developed for detecting $C$ psittaci in tissue sections $^{8}$ (Figs. 4 and 5) This method does not, however, differentiate between $C$ psittaci and $C$ trachomatis. Nevertheless, no glycogen matrix within the cytoplasmic inclusions could be identified in preparations stained with Lugol's iodine, periodic acid Schiff, or Best's carmine, suggesting that they were unlikely to be $C$ trachomatis organisms.

The ultrastructural appearance of the cytoplasmic inclusions was investigated by transmission electron microscopy (Figs. 6-8) and compared with previously published descriptions of chlamydiae in cells. $^{9-12}$ The inclusions were usually surrounded by membrane and were densely packed with structures which had the appearance of chlamydial infectious elementary bodies, with diameters ranging from 300 to $500 \mathrm{~nm}$. In addition, occasional structures characteristic of chlamydial reticulate and intermediate bodies were present. Each of these chlamydial bodies was enclosed by a rigid envelope representing the cell wall. No glycogen matrix could be found.

\section{Discussion}

The cultural and serological data, together with the histopathological, immunohistochemical, ${ }^{8}$ and ultrastructural findings, indicate that this placentitis was associated with infection by $C$ psittaci, which might 
well have been acquired from the aborting ewes which the patient had handled during recent lambing. No attempt was made to ascertain the cause of lamb loss at the time, but subsequent investigation showed that $C$ psittaci were cultured from the faeces of the affected ewes. It would therefore seem probable that infection with the organism was the cause of the initial influenza like illness and the subsequent spontaneous abortion and disseminated intravascular coagulation. The histological pattern of the placentitis suggests a haematogenous spread of the infection from the maternal circulation, rather than infection by an ascending cervical route. The primary site of maternal infection or carriage was not identified; there was, however, no clinical or radiological evidence of any pneumonic process.

While $C$ trachomatis is recognised more frequently as a cause of a variety of diseases, especially genital infections, ${ }^{1314}$ it is pertinent that $C$ psittaci is widespread in this country and economically important to the farming community. Between 1980 and 1981 in more than 1000 farms in the UK lambing losses due to chlamydial infections were as great as $30 \%$, a figure which appears to be increasing. ${ }^{\prime}$ Sporadic cases of human abortion in these areas will occur, and we hope that this report will alert the clinician and pathologist to the possibility that $C$ psittaci may be a cause of human placentitis and subsequent abortion. The diagnosis can be made even on formalin fixed material by the relatively simple methods outlined in this report.

We are grateful to Dr M Moffat for alerting us to the first clue to the aetiology in this case and Professor $R$ Postlethwaite for his helpful comments. We also thank the technical staff of the Department of Pathology, University of Aberdeen, in particular Mr
A McKinnon, Mrs L Doverty, Miss S Cockburn, and Mrs M Taylor, for expert assistance.

\section{References}

' Johnson FWA. Zoonosis in practice-chlamydiosis. Br Vet J 1983;139:93-101.

${ }^{2}$ Beer RJS, Bradford WP, Hart RJC. Pregnancy complicated by psittacosis acquired from sheep. Br Med J 1982:284: 1156-7.

${ }^{3}$ Hobson D, Rees E. Pregnancy complicated by psittacosis acquired from sheep. Br Med J 1982;284:1560-1.

4 Roberts W, Grist NE, Giroud P. Human abortion associated with infection by ovine abortion agent. $\mathrm{Br}$ Med J 1967;iv: 37 .

s Schachter J. Isolation of Bedsoniae from human arthritis and abortion tissue. Am J Ophthalmol 1967;63:1082-6.

- Johnson FWA, Matheson BA, Williams H, et al. Abortion due to infection with Chlamydia psittaci in a sheep farmer's wife. $\mathrm{Br}$ Med J 1985; 290:592-4.

' Johnson FWA, Chancerelle LYJ, Hobson D. An improved method for demonstrating the growth of Chlamydiae in tissue culture. Med Lab Sci 1978;35:67-74.

${ }^{8}$ Finlayson J, Buxton D, Anderson I, Donald K. A direct immunoperoxidase method for demonstrating Chlamydia psittaci in tissue sections. J Clin Pathol 1985;38:712-4.

- Tamura A, Matsumoto A, Mariene GP, Higashi N. Electron microscopic observations on the structure of the envelopes of matured elementary bodies and developmental reticulate forms of Chlamydia psittaci. J Bacteriol 1971;105:355-60.

${ }^{10}$ Swanson J, Eschenbach DA, Alexander ER, Holmes KK. Light and electron microscopic studies of Chlamydia trachomatis infection of the uterine cervix. J Infect Dis 1975;131:678-87.

" Higashi N. Electron microscopic studies on the mode of reproduction of trachoma virus and psittacosis virus in cell cultures. J Exp Mol Pathol 1965;4:24-39.

12 Orfila FEBJ, Lefevre JF. Ultrastructural studies of the development of the agents of ewe's abortion. $J$ Ultrastructure 1976; 56: 177-85.

${ }^{13}$ Taylor-Robinson D, Thomas BJ. The role of Chlamydia trachomatis in genital tract and associated diseases. J Clin Pathol 1980;33:205-33.

${ }^{14}$ Oriel JD, Ridgway GL. Current topics in infection, Vol 2. London: Arnold, 1982:41-66.

Requests for reprints to: Dr SY Wong, Department of Pathology, University Medical Buildings, Foresterhill, Aberdeen AB9 2ZD, Scotland. 